\title{
SUBHARMONIC SOLUTIONS OF CONSERVATIVE SYSTEMS WITH NONCONVEX POTENTIALS
}

\author{
A. FONDA AND A. C. LAZER
}

(Communicated by Kenneth R. Meyer)

\begin{abstract}
We consider the system of second order differential equations

$$
u^{\prime \prime}+\nabla G(u)=e(t) \equiv e(t+T)
$$

where the potential $G: \mathbb{R}^{n} \rightarrow \mathbb{R}$ is not necessarily convex. Using critical point theory, we give conditions under which the system has infinitely many subharmonic solutions.
\end{abstract}

\section{INTRODUCTION}

In this note, we consider the following system of second order differential equations:

$$
u^{\prime \prime}+\nabla G(u)=e(t) .
$$

Here, $G: \mathbb{R}^{n} \rightarrow \mathbb{R}$ is a continuously differentiable function with gradient $\nabla G$ and $e: \mathbb{R} \rightarrow \mathbb{R}^{n}$ is a continuous periodic function having a minimal period $T>0$. We are interested in the existence of subharmonic solutions of (1), i.e., of periodic solutions of (1) having minimal period in the set $\{k T: k \in \mathbb{N}, k \geq$ $2\}$. The existence of this type of solutions is not always guaranteed, as the case $G \equiv 0$ shows. Indeed, in this case, every periodic solution of (1) has minimal period $T$ (see [8]).

There have been various types of results concerning the existence of subharmonic solutions to systems like (1) or to more general first order Hamiltonian systems. These have been obtained either by perturbation techniques $[1,2]$ or, starting with [12], by some global approach. Most of the results proving the existence of subharmonic solutions in the above sense, however, have made use of a convexity assumption on the potential $[3,6,7,10,11,14-16]$, or else some "generic" type results were proved $[4,8]$. For $n=1$, the case of superlinear nonlinearities has been studied in $[5,9]$ by phase-plane methods.

Here we prove the existence of subharmonic solutions without assuming the convexity of $G$ by simply making some careful estimates on the critical levels of the functionals associated to the problem.

Received by the editors November 23, 1990.

1980 Mathematics Subject Classification (1985 Revision). Primary 34C25; Secondary 58E05.

Key words and phrases. Critical point, Saddle Point Theorem, Palais-Smale condition. 


\section{THE MAIN RESULT}

Our main result concerning the system (1) is the following one, where we denote the mean value of $e(t)$ by $\bar{e}$, i.e.,

$$
\bar{e}=\frac{1}{T} \int_{0}^{T} e(t) d t
$$

Theorem 1. Assume that the range of $\nabla G$ is bounded,

(i) $\exists M>0: \forall u \in \mathbb{R}^{n},\|\nabla G(u)\| \leq M$.

If moreover

(ii) $\lim _{\|u\| \rightarrow \infty}\langle\nabla G(u)-\bar{e}, u\rangle=+\infty$,

then system (1), besides having at least one $T$-periodic solution, also has periodic solutions with minimal period $k T$, for any sufficiently large prime number $k$.

In the proof of Theorem 1 we will consider, for every positive integer $k$, the continuously differentiable functional

$$
\varphi_{k}(u)=\int_{0}^{k T}\left\{\frac{1}{2}\left\|u^{\prime}\right\|^{2}-G(u)+\langle e, u\rangle\right\} d t,
$$

defined on the space $H_{k T}^{1}$ of $k T$-periodic absolutely continuous vector functions whose derivatives have square-integrable norm. We will denote the usual norm in $H_{k T}^{1}$ by $\|\cdot\|_{k T}$. One has

$$
\left.\varphi_{k}^{\prime}(u) v=\int_{0}^{k T}\left\{\left\langle u^{\prime}, v^{\prime}\right\rangle-\langle\nabla G(u), v\rangle+\langle e, v\rangle\right\rangle\right\} d t,
$$

and it is well known that the critical points of the functional $\varphi_{k}$ correspond to the $k T$-periodic solutions of the system (1) (cf. [10,13].

In the first step, we will show that the set $S_{T}$ of $T$-periodic solutions of (1) is bounded in $H_{T}^{1}$. As a consequence, $\varphi_{1}\left(S_{T}\right)$ is bounded, and, since for any $u \in S_{T}$ one has $\varphi_{k}(u)=k \varphi_{1}(u)$, we have

$$
\exists c>0: \forall u \in S_{T}, \quad \forall k \geq 1, \frac{1}{k}\left|\varphi_{k}(u)\right| \leq c .
$$

Next, we will show that, for every positive integer $k$, one can find a $k T$ periodic solution $u_{k}$ of (1) in such a way that the sequence $\left(u_{k}\right)$ has the property

$$
\lim _{k \rightarrow \infty} \frac{1}{k} \varphi_{k}\left(u_{k}\right)=-\infty
$$

This will be done by the use of some estimates on the critical levels of $\varphi_{k}$ given by the Saddle Point Theorem of Rabinowitz. Consequently, for $k$ large enough, $u_{k} \notin S_{T}$, and if $k$ is chosen to be a prime number, the minimal period of $u_{k}$ has to be $k T$.

The following lemma will be needed for the study of the geometry of the functionals $\varphi_{k}$. It also shows that Theorem 1 improves a result in [7] obtained for systems like (1) with a convex potential $G$ by the use of Morse theory.

Lemma 1. If the assumptions of Theorem 1 hold, then

(ii') $\lim _{\|u\| \rightarrow \infty}(G(u)-\langle\bar{e}, u\rangle)=+\infty$. 
Moreover, condition (ii) happens to be equivalent to (ii') whenever $G$ is assumed to be convex.

Proof. Assume (i) and (ii) in Theorem 1. Let $R>0$ be such that

$$
\|u\| \geq R \Rightarrow\langle\nabla G(u)-\bar{e}, u\rangle \geq 1 .
$$

Then, for $\|u\| \geq R$, we have

$$
\begin{aligned}
G(u)-\langle\bar{e}, u\rangle & =G(0)+\int_{0}^{R /\|u\|}\langle\nabla G(s u)-\bar{e}, u\rangle d s+\int_{R /\|u\|}^{1}\langle\nabla G(s u)-\bar{e}, u\rangle d s \\
& \geq G(0)-(M+\|\bar{e}\|) R+\int_{R /\|u\|}^{1} \frac{1}{s} d s \\
& =G(0)-(M+\|\bar{e}\|) R+\log \left(\frac{\|u\|}{R}\right),
\end{aligned}
$$

and (ii ${ }^{\prime}$ ) follows.

Now assume $G$ to be convex. Then, for every $x, y \in \mathbb{R}^{n}$, we have

$$
G(y) \geq G(x)+\langle\nabla G(x), y-x\rangle
$$

(cf. [10]). Choosing $y=0$ shows that (ii') implies (ii). On the other hand, if (ii) holds, we can choose $R>0$ for which (3) is satisfied. Using (4) again, we get:

$$
\begin{aligned}
G(u)-\langle\bar{e}, u\rangle \geq & G\left(\frac{R}{\|u\|} u\right)-\left\langle\bar{e}, \frac{R}{\|u\|} u\right\rangle \\
& +\left(\frac{\|u\|}{R}-1\right)\left\langle\nabla G\left(\frac{R}{\|u\|} u\right)-\bar{e}, \frac{R}{\|u\|} u\right\rangle \\
\geq & \frac{\|u\|}{R}-1+\min _{\|v\|=R}\{G(v)-\langle\bar{e}, v\rangle\},
\end{aligned}
$$

and (ii') follows immediately.

Proof of Theorem 1. First of all, we notice that it is of no loss of generality to suppose that $\bar{e}=0$ and, in view of Lemma 1 , that $G(u) \geq 0$ for every $u$. Hence we will assume this throughout the proof. As we explained above, we begin the proof by showing that the set $S_{T}$ of $T$-periodic solutions of (1) is bounded in $H_{T}^{1}$. Assume by contradiction that there exists a sequence $\left(u_{n}\right)$ in $S_{T}$ such that $\left\|u_{n}\right\|_{T} \rightarrow \infty$. Let us write $u_{n}(t)=\bar{u}_{n}+\widetilde{u}_{n}(t)$, where $\bar{u}_{n}$ is the mean value of $u_{n}$. Multiplying both sides of the identity

$$
u_{n}^{\prime \prime}(t)+\nabla G\left(u_{n}(t)\right)=e(t)
$$

by $\tilde{u}_{n}(t)$ and integrating, we obtain

$$
-\int_{0}^{T}\left\|\tilde{u}_{n}^{\prime}\right\|^{2}+\int_{0}^{T}\left\langle\nabla G\left(u_{n}\right), \tilde{u}_{n}\right\rangle=\int_{0}^{T}\left\langle e, \tilde{u}_{n}\right\rangle .
$$

Using assumption (i), we easily deduce that $\left(\widetilde{u}_{n}\right)$ is bounded in $H_{T}^{1}$. Hence,

$$
\min _{0 \leq t \leq T}\left\|u_{n}(t)\right\| \rightarrow \infty .
$$

Multiplying (5) by $u_{n}$ and integrating, we get

$$
-\int_{0}^{t}\left\|\tilde{u}_{n}^{\prime}\right\|^{2}+\int_{0}^{t}\left\langle\nabla G\left(u_{n}\right), u_{n}\right\rangle=\int_{0}^{t}\left\langle e, \tilde{u}_{n}\right\rangle .
$$


Now, since $\left(\widetilde{u}_{n}\right)$ is bounded in $H_{T}^{1}$, we deduce from (7) that

$$
\left(\int_{0}^{T}\left\langle\nabla G\left(u_{n}\right), u_{n}\right\rangle\right)
$$

is bounded, but this is in contradiction with (6) and assumption (ii).

Now we need to show that, for every positive integer $k$, one can find a critical point $u_{k}$ of the functional $\varphi_{k}$ in such a way that (2) holds. To this aim, we will apply the Saddle Point Theorem (cf. [13, Theorem 4.6]) to each of the $\varphi_{k}$ 's. Let us fix $k$ and write $H_{k T}^{1}=\mathbb{R}^{n} \oplus \widetilde{H}_{k T}$, where $\mathbb{R}^{n}$ is identified with the set of constant functions and $\widetilde{H}_{k T}$ consists of functions $\widetilde{u}$ in $H_{k T}^{1}$ such that $\int_{0}^{k T} \tilde{u}(s) d s=0$. First, we prove the Palais-Smale condition. Let $\left(u_{n}\right)$ be a consequence in $H_{k T}^{1}$ such that $\varphi_{k}\left(u_{n}\right)$ is bounded and $\varphi_{k}^{\prime}\left(u_{n}\right) \rightarrow 0$ as $n \rightarrow \infty$. In particular, for a positive constant $c_{1}$, we will have

$$
\varphi_{k}^{\prime}\left(u_{n}\right) \tilde{u}_{n}=\int_{0}^{k T}\left\{\left\|\tilde{u}_{n}^{\prime}\right\|^{2}-\left\langle\nabla G\left(u_{n}\right), \tilde{u}_{n}\right\rangle+\left\langle e, \tilde{u}_{n}\right\rangle\right\} d t \leq c_{1}\left\|\tilde{u}_{n}\right\|_{k T} .
$$

Then it follows from (i) that $\left(\widetilde{u}_{n}\right)$ is bounded in $H_{k T}^{1}$. Since

$$
\varphi_{k}\left(u_{n}\right)=\int_{0}^{k T}\left\{\frac{1}{2}\left\|\widetilde{u}_{n}^{\prime}\right\|^{2}-G\left(u_{n}\right)+\left\langle e, \widetilde{u}_{n}\right\rangle\right\} d t
$$

is bounded, it then follows from the uniform boundedness of $\left(\widetilde{u}_{n}\right)$ and from $\left(i i^{\prime}\right)$ in Lemma 1 that $\left(\bar{u}_{n}\right)$ has to be bounded too. So $\left(u_{n}\right)$ is bounded in $H_{k T}^{1}$, and it is now a standard argument to show that $\left(u_{n}\right)$ has a convergent subsequence (see [13, Appendix B]). Hence, the Palais-Smale condition holds.

It is easy to show that (i) yields the coercivity of $\varphi_{k}$ on $\widetilde{H}_{k T}$, while (ii), through (ii') of Lemma 1 , yields the coercivity of $\left(-\varphi_{k}\right)$ on $\mathbb{R}^{n}$. For $r>0$, let us denote by $D_{r}$ the closed disc in ${ }^{n}$ centered in 0 with radius $r$, and by $\partial D_{r}$ its boundary. It follows from the above that, for a sufficiently large $r_{k}$, one has

$$
\inf _{H_{k T}} \varphi_{k}>\max _{D_{r_{k}}} \varphi_{k} .
$$

so the assumptions of the Saddle Point Theorem are all satisfied, and we can find a critical point $x_{k}$ of $\varphi_{k}$ such that

$$
\varphi_{k}\left(x_{k}\right)=\inf _{\gamma \in \Gamma_{k}} \max _{\xi \in D_{r_{k}}} \varphi_{k}(\gamma(\xi)),
$$

where $\Gamma_{k}=\left\{\gamma \in C\left(D_{r_{k}}, H_{k T}^{1}\right): \gamma=\right.$ id on $\left.\partial D_{r_{k}}\right\}$. In particular, $\varphi_{1}$ has a critical point, i.e., (1) has a $T$-periodic solution.

In order to prove that the sequence $\left(x_{k}\right)$ satisfies $(2)$, we will show that for every $m>0$ there exists a positive integer $\bar{k}$ such that, for each $k \geq \bar{k}$, we can construct $\gamma_{k} \in C\left(D_{r_{k}}, H_{k T}^{1}\right)$ with the property that

$$
\frac{1}{k} \max _{\xi \in D_{r_{k}}} \varphi_{k}\left(\gamma_{k}(\xi)\right) \leq-m \text {. }
$$

First of all, we notice that $r_{k}$ above can be taken such that

$$
r_{k} \geq \sqrt{n} \cdot k
$$


Let us fix $m>0$. using (ii') of Lemma 1 , take $\bar{k}$ such that

$$
\|x\| \geq \bar{k} \Rightarrow G(x) \geq\left(3 m T+24 n \pi^{2}\right) / T^{2} .
$$

For $k \geq \bar{k}$, we construct $\gamma_{k} \in C\left(D_{r_{k}}, H_{k T}^{1}\right)$ as follows:

$$
\gamma_{k}(\xi)(t)=\xi+\left(1-\|\xi\| / r_{k}\right) w_{k}(t),
$$

where $w_{k}(t)$ is the vector function $w_{k}(t)=\left(w_{k}^{1}(t), \ldots, w_{k}^{n}(t)\right)$ with $w_{k}^{i}(t)=$ $2 k \sin (2 \pi t / k T), i=1, \ldots, n$. Then we have

$$
\begin{aligned}
\frac{1}{k} \max _{\xi \in D_{r_{k}}} \varphi_{k}\left(\gamma_{k}(\xi)\right)=\frac{1}{k} \max _{\xi \in D_{r_{k}}} \int_{0}^{k T}\left\{\frac{1}{2}\left[\left(1-\frac{\|\xi\|}{r_{k}}\right) 2 k \sqrt{n} \frac{2 \pi}{k T} \mid \cos \left(\frac{2 \pi}{k T} t\right)\right]^{2}\right. \\
\left.-G\left(\gamma_{k}(\xi)(t)\right)+\left\langle e(t), \gamma_{k}(\xi)(t)\right\rangle\right\} d t \\
\leq \frac{8 n \pi 2}{T}-\min _{\xi \in D_{r_{k}}} \frac{T}{2 \pi} \int_{0}^{2 \pi} G\left(\xi+\left(1-\frac{\|\xi\|}{r_{k}}\right) w_{k}\left(\frac{k T}{2 \pi} s\right)\right) d s,
\end{aligned}
$$

since, by a Fourier series argument

$$
\int_{0}^{k T}\left\langle e(t), \gamma_{k}(\xi)(t)\right\rangle d t=0
$$

We claim that, for every $\xi \in D_{r_{k}}$ one has

$$
\left\|\xi+\left(1-\frac{\|\xi\|}{r_{k}}\right) w_{k}\left(\frac{k T}{2 \pi} s\right)\right\| \geq k
$$

on a subset of $[0,2 \pi]$ having measure of at least $\left(\frac{2}{3} \pi\right)$. Let $\bar{i}$ be such that $\left|\xi_{\bar{i}}\right|=\max \left\{\left|\xi_{i}\right|, i=1, \ldots, n\right\}$. If $\xi_{\bar{i}}>0$, for every $s \in\left[\frac{1}{6} \pi, \frac{5}{6} \pi\right]$, we have

$$
\begin{aligned}
\left\|\xi+\left(1-\frac{\|\xi\|}{r_{k}}\right) w_{k}\left(\frac{k T}{2 \pi} s\right)\right\| & \geq\left|\xi_{\bar{i}}+\left(1-\frac{\|\xi\|}{r_{k}}\right) 2 k \sin s\right| \\
& =\left|\frac{\|\xi\|}{r_{k}}\left(\frac{r_{k}}{\|\xi\|} \xi_{\bar{i}}\right)+\left(1-\frac{\|\xi\|}{r_{k}}\right) 2 k \sin s\right| \geq k,
\end{aligned}
$$

since $\left(r_{k} \xi_{\bar{i}} /\|\xi\|\right) \geq k$ by $(8)$ and $2 k$ sin $s \geq k$ for $s \in\left[\frac{1}{6} \pi, \frac{5}{6} \pi\right]$. With a similar computation, one can see that, whenever $\xi_{\bar{i}}<0$, (11) holds for every $s \in\left[\frac{7}{6} \pi, \frac{11}{6} \pi\right]$. In the case $\xi_{\bar{i}}=0$, i.e., $\xi=0$, one has (11) for both $s \in$ $\left[\frac{1}{6} \pi, \frac{5}{6} \pi\right]$ and $s \in\left[\frac{7}{6} \pi, \frac{11}{6} \pi\right]$, proving the claim.

Using (9) and the fact that $G$ is supposed to be nonnegative, from (10) we get

$$
\frac{1}{k} \max _{\xi \in D_{r_{k}}} \varphi_{k}\left(\gamma_{k}(\xi)\right) \leq \frac{8 n \pi^{2}}{T}-\frac{T}{2 \pi}\left(\frac{2}{3} \pi\right) \frac{3 m T+24 n \pi^{2}}{T^{2}}=-m,
$$

and the proof is complete.

\section{THE CASE $\mathbf{N}=1$}

When dealing with the scalar equation

$$
u^{\prime \prime}+g(u)=e(t)
$$

we are able to prove a result with less restrictive assumptions on the nonlinearity than those required in Theorem 1.

We set $G(u)=\int_{0}^{u} g(x) d x$ and, as before, $\bar{e}=\frac{1}{T} \int_{0}^{T} e(t) d t$. 
Theorem 2. Assume the following conditions:

(j) $\lim _{|u| \rightarrow \infty} \sup g(u) / u<(2 \pi / T)^{2}$;

(jj) $\exists d>0: \forall|u| \geq d,(g(u)-\bar{e}) u>0$;

(jjj) $\lim _{|u| \rightarrow \infty} 2 G(u) / u^{2}=0$;

(jv) $\lim _{|u| \rightarrow \infty}(G(u)-\bar{e} u)=+\infty$.

Then equation (12), besides having at least one $T$-periodic solution, also has periodic solutions with minimal period $k T$, for any sufficiently large prime integer $k$.

Proof. We follow the same ideas of the proof of Theorem 1. Again we can assume without loss of generality that $\bar{e}=0$ and $G(u) \geq 0$ for every $u \in \mathbb{R}$. In order to prove the boundedness of the set of $T$-periodic solutions we argue by contradiction. Assume that there exists a sequence $\left(u_{n}\right)$ in $S_{T}$ such that $\left\|u_{n}\right\|_{T} \rightarrow \infty$. By $(\mathrm{j})$ and $(\mathrm{jj})$, we can write

$$
g(u)=g_{0}(u) u+g_{1}(u),
$$

where $0 \leq g_{0}(u) \leq(2 \pi / T)^{2}-\delta$ for some $\delta>0$ and every $u \in \mathbb{R}$ and the range of $g_{1}$ is bounded. Setting $v_{n}=u_{n} /\left\|u_{n}\right\|_{T}$, we have

$$
v_{n}^{\prime \prime}+g_{0}\left(u_{n}\right) v_{n}+\frac{g_{1}\left(u_{n}\right)}{\left\|u_{n}\right\|_{T}}=\frac{e(t)}{\left\|u_{n}\right\|_{T}}
$$

Hence, $\left(v_{n}\right)$ is bounded in $C^{2}$, and a subsequence converges strongly in $C^{1}$ to a certain map $v$. The sequence $\left(g_{0}\left(u_{n}\right)\right)$ converges weakly in $L^{2}$ to some $\alpha(t)$ that, by the weak closure of the convex set $\left\{f \in L^{2}: 0 \leq f(t) \leq(2 \pi / T)^{2}-\right.$ $\delta$, a.e., $t\}$, satisfies

$$
0 \leq \alpha(t) \leq(2 \pi / T)^{2}-\delta
$$

for almost every $t$. Passing to the weak limit in (13) we get

$$
v^{\prime \prime}+\alpha(t) v=0
$$

Since $\|v\|_{T}=1$, it follows from (14) and (15) that $\alpha \equiv 0$. Hence, $v$ is a constant, and $\left(u_{n}\right)$ is such that

$$
\min _{0 \leq t \leq T}\left|u_{n}(t)\right| \rightarrow \infty
$$

but, integrating equation (12) gives

$$
\int_{0}^{T} g\left(u_{n}(t)\right) d t=0
$$

which is in contradiction with (jj) and (16).

Let us prove the Palais-Smale condition for the functionals $\varphi_{k}$. Fix $k$, and write $H_{k T}^{1}=\mathbb{R} \oplus \widetilde{H}_{k T}$. Let $\left(u_{n}\right)$ be a sequence in $H_{k T}^{1}$ such that $\varphi_{k}\left(u_{n}\right)$ is bounded and $\varphi_{k}^{\prime}\left(u_{n}\right) \rightarrow 0$ as $n \rightarrow \infty$. Assume by contradiction that, for a subsequence, $\left\|u_{n}\right\|_{k T} \rightarrow \infty$. First we prove that, as $n \rightarrow \infty,\left|\bar{u}_{n}\right| \rightarrow \infty$ and

$$
\frac{\left\|\widetilde{u}_{n}\right\|_{k T}}{\left|\bar{u}_{n}\right|} \rightarrow 0
$$

Fix $\varepsilon>0$ sufficiently small. Condition (jjj) implies that there is a $c_{\varepsilon}>0$ such that

$$
G(u) \leq \frac{\varepsilon}{8 k T} u^{2}+c_{\varepsilon}
$$


for every $u \in \mathbb{R}$. Then,

$$
\begin{aligned}
\varphi_{k}\left(u_{n}\right) \geq & \frac{1}{2} \int_{0}^{k T}\left(\widetilde{u}_{n}^{\prime}\right)^{2}-\frac{\varepsilon}{8 k T} \int_{0}^{k T} \widetilde{u}_{n}^{2}-\frac{\varepsilon}{8} \bar{u}_{n}^{2} \\
& -k T c_{\varepsilon}-\left(\int_{0}^{k T} e^{2}\right)^{1 / 2}\left(\int_{0}^{k T} \widetilde{u}_{n}^{2}\right)^{1 / 2} \\
\geq & \frac{1}{4} \int_{0}^{k T}\left(\tilde{u}_{n}^{\prime}\right)^{2}-\frac{\varepsilon}{8} \bar{u}_{n}^{2}-c_{\varepsilon}{ }^{\prime}
\end{aligned}
$$

for some constant $c_{\varepsilon}^{\prime}>0$. We have used the Wirtinger inequality, assuming that $\varepsilon$ is small enough. Since $\varphi_{k}\left(u_{n}\right)$ is bounded, it follows that $\left|\bar{u}_{n}\right| \rightarrow \infty$, since otherwise, for a subsequence, $\left(\left|\bar{u}_{n}\right|\right)$ would be bounded and, by (18), $\left(\tilde{u}_{n}^{\prime}\right)$ would be bounded too, contradicting the fact that $\left\|u_{n}\right\|_{k T} \rightarrow \infty$. Moreover, multiplying (18) by $\left(1 / \bar{u}_{n}^{2}\right)$, one can find a constant $c_{\varepsilon}^{\prime \prime}>0$ such that

$$
\frac{\left(\int_{0}^{k T}\left(\tilde{u}_{n}^{\prime}\right)^{2}\right)}{\tilde{u}_{n}^{2}} \leq \frac{\varepsilon}{2}+\frac{c_{\varepsilon}^{\prime \prime}}{\bar{u}_{n}^{2}} \leq \varepsilon,
$$

for $n$ sufficiently large. Hence, we proved that

$$
\lim _{n \rightarrow \infty} \frac{\int_{0}^{k T}\left(\widetilde{u}_{n}^{\prime}\right)^{2}}{\bar{u}_{n^{2}}}=0 .
$$

By the Wirtinger inequality, (17) easily follows. Using the Sobolev inequality, we can deduce from (17) that

$$
\min _{0 \leq t \leq T}\left|u_{n}(t)\right| \rightarrow \infty,
$$

as $n \rightarrow \infty$. Since $\varphi_{k}^{\prime}\left(u_{n}\right) \rightarrow 0$, there exists a constant $c_{1}$ such that $\left|\varphi_{k}\left(u_{n}\right) h\right| \leq$ $c_{1}\|h\|_{k T}$ for every $h \in H_{k T}^{1}$. In particular, taking $h \equiv 1$, we get

$$
\left|\int_{0}^{k T} g\left(u_{n}(t)\right) d t\right| \leq c_{1} k T
$$

while, taking $h=\widetilde{u}_{n}$, we have

$$
\left|\int_{0}^{k T}\left\{\tilde{u}_{n}^{\prime 2}-g\left(u_{n}\right) \tilde{u}_{n}+e \widetilde{u}_{n}\right\} d t\right| \leq c_{1}\left\|\widetilde{u}_{n}\right\|_{k T} .
$$

Using (19) and (jj), for $n$ large enough, one has

$$
\left|\int_{0}^{k T} g\left(u_{n}(t)\right) d t\right|=\int_{0}^{k T}\left|g\left(u_{n}(t)\right)\right| d t,
$$

and from (20) and (21) we have that $\left(\tilde{u}_{n}\right)$ is bounded. $\varphi_{k}\left(u_{n}\right)$ being bounded, we conclude that $\left(\int_{0}^{k T} G\left(u_{n}(t)\right) d t\right)$ has to be bounded too, but this is in contradiction to (19) and (jv). Hence, the sequence $\left(u_{n}\right)$ has to be bounded, and the Palais-Smale condition holds.

The geometry of the functionals $\varphi_{k}$ are easily handled through conditions (jjj) and (jv), showing that we are in the hypothesis of the Saddle Point Theorem. Finally, one can use, and indeed simplify, the argument ending the proof of the 
Theorem 1 to prove that $(2)$ holds for the sequence $\left(u_{k}\right)$ of critical points found in this way.

Theorem 2 improves a result in [7] where the function $g$ was supposed to be increasing. As an easy consequence, we have the following

Corollary. Assume that

(j') $\lim _{|u| \rightarrow \infty} g(u) / u=0$,

$\left(\mathrm{jj}^{\prime}\right) \lim _{|u| \rightarrow \infty} \inf (g(u)-\bar{e}) u>0$

Then the conclusion of Theorem 2 holds.

\section{REFERENCES}

1. V. Benci and D. Fortunato, A Birkhoff-Lewis type result for a class of Hamiltonian systems Manuscripta Math. 59 (1987), 441-456.

2. G. D. Birkhoff and D. C. Lewis, On the periodic motions near a given periodic motion of a dynamical system, Ann. Mat. Pura Appl. 12 (1933), 117-133.

3. F. Clarke and I. Ekeland, Nonlinear oscillations and boundary value problems for Hamiltonian systems, Arch. Rational Mech. Anal. 78 (1982), 315-333.

4. C. Conley and E. Zehnder, Subharmonic solutions and Morse theory, Physica A 124 (1984), 649-658.

5. T. Ding and F. Zanolin, Periodic solutions of Duffing's equations with superquadratic potential, preprint.

6. I. Ekeland and H. Hofer, Subharmonics for convex nonautonomous Hamiltonian systems, Comm. Pure Appl. Math. 40 (1987), 1-36.

7. A. Fonda, M. Ramos, and M. Willem, Subharmonic solutions for second order differential equations, preprint.

8. A. Fonda and M. Willem, Subharmonic oscillations of forced pendulum-type equations, J. Differential Equations 81 (1989), 215-220.

9. H. Jacobowitz, Periodic solutions of $x^{\prime \prime}+f(x, t)=0$ via the Poincaré-Birkhoff Theorem, J. Differential Equations 29 (1976), 37-52.

10. J. Mawhin and M. Willem, Critical point theory and hamiltonian systems, Springer-Verlag, New York, 1988.

11. R. Michalek and G. Tarantello, Subharmonic solutions with prescribed minimal period for nonautonomous Hamiltonian systems, J. Differential Equations 72 (1988), 28-55.

12. P. H. Rabinowitz, On subharmonic solutions of Hamiltonian systems, Comm. Pure Appl. Math. 33 (1980), 609-633.

13. _ Minimax methods in critical point theory with applications to differential equations, CBMS Reg. Conf. Ser. in Math. no. 65, Amer. Math. Soc., Providence, RI, 1986.

14. G. Tarantello, Subharmonic solutions for Hamiltonian systems via $\mathbb{Z}_{p}$-pseudo index theory, preprint.

15. M. Willem, Subharmonic oscillations of convex Hamiltonian systems, Nonlin. Anal. 9 (1985), 1303-1311.

16. Z. Yang, The existence of subharmonic solutions for sublinear Duffing's equation, preprint.

Institut de Mathematique Pure et Appliquee, Universite Catholique de louvain, Chemin du Cyclotron 2, B-1348 Louvain-Le-Neuve, Belgium

Department of Mathematics and Computer Science, University of Miami, Coral GABLES, FLORIDA 33124 